\title{
Sponges of the family Esperiopsidae (Demospongiae, Poecilosclerida) from Northwest Africa, with the descriptions of four new species
}

\author{
Rob W.M. Van Soest, Elly J. Beglinger \& Nicole J. de Voogd \\ Naturalis Biodiversity Center, Darwinweg 2, 2333 CR, Leiden, The Netherlands \\ Email: rob.vansoest@,naturalis.nl
}

\begin{abstract}
Sponges belonging to the genera Amphilectus Vosmaer, Esperiopsis Carter and Ulosa de Laubenfels of the family Esperiopsidae were collected during 1986 and 1988 expeditions of the Netherlands Centre for Biodiversity Naturalis (at that time the National Museum of Natural History at Leiden and the Zoological Museum of Amsterdam) in waters off the coasts of Mauritania and the Cape Verde Islands. Four new species, Amphilectus utriculus sp. nov., Amphilectus strepsichelifer sp. nov., Esperiopsis cimensis sp. nov., Ulosa capblancensis sp. nov., and two already known species, Amphilectus cf. fucorum (Esper) and Ulosa stuposa (Esper) are described and discussed.
\end{abstract}

Keywords. Sponges, new species, Esperiopsidae, Mauritania, Cape Verde Islands.

Van Soest R.W.M., Beglinger E.J. \& de Voogd N.J. 2012. Sponges of the family Esperiopsidae (Demospongiae, Poecilosclerida) from Northwest Africa, with the descriptions of four new species. European Journal of Taxonomy 18: 1-21. http://dx.doi.org/10.5852/ejt.2012.18

\section{Introduction}

Knowledge of the sponge fauna of Northwest Africa is poor, with few recent publications and older papers usually based on specimens obtained during wide-ranging expeditions with often only summary descriptions (Lendenfeld 1907; Topsent 1918, 1928; Arnesen 1932; Burton 1956; Lévi 1952, 1956, 1959, 1960; Van Soest 1993a, b). Preliminary analysis (Van Soest 1993a) suggested that the main affinity of the sponge fauna of Northwest Africa lies with the Lusitanian and Western Mediterranean faunas, with minor affinity with the Tropical Western Atlantic, and a considerable endemic element. Expeditions made in the 1980's (CANCAP 1-7; Mauritania II) yielded ample materials from the coasts of Mauritania, the Canary and Madeira Archipelagoes and the Cape Verde Islands, incorporated in the collections of the former Rijksmuseum van Natuurlijke Historie at Leiden and the Zoölogisch Museum Amsterdam, recently merged into the collections of the Netherlands Centre for Biodiversity Naturalis at Leiden. Sponges were collected by various methods (wading, snorkeling, SCUBA, Van Veen grab, Agassiz trawls and rectangular dredges) from shallow water down to $>1000 \mathrm{~m}$. This material was supplemented by specimens, previously collected by F.P. Vermeulen in Mauritanian and Senegalese waters. The combined collections contain several sponges new to science and many interesting new finds of ill-known sponges. A number of scientific studies containing descriptions of sponges from these Northwest African collections preceded the present one, e.g. on Haplosclerida Topsent, 1928 (De Weerdt \& Van Soest 1986), the genera Didiscus Dendy, 1922 (Hiemstra \& Van Soest 1991), Acarnus Gray, 1867 (Van Soest et al. 1991), Hemiasterella Carter, 1879 (Voultsiadou-Koukoura \& Van Soest 
1991), and Dercitus Gray, 1867 (Van Soest et al. 2010). The present paper addresses sponges of the poecilosclerid family Esperiopsidae Hentschel, 1923 collected off the coasts of Mauritania and the Cape Verde Islands. The family contains four accepted genera (Van Soest \& Hajdu 2002b), three of which appear to have representatives in Northwest African waters, viz. Amphilectus Vosmaer, 1880, Esperiopsis Carter, 1882, and Ulosa de Laubenfels, 1936. Only the genus Semisuberites Carter, 1877 has so far not been recorded. The three genera present in the material are easily distinguished, possibly because they are 'artificial', but this remains unchallenged as no comprehensive phylogenetic research has been done on members of this group. Skeletons consist of reticulated bundles of styles, there is no special surface arrangement of spicules, microscleres combine the presence of chelae and sigmas (Esperiopsis), consist of chelae only (Amphilectus), or are absent (Ulosa). Whereas three genera tend to have cold-water affinity (Van Soest et al. 2012), at least Ulosa appears to have also tropical or warmwater representatives.

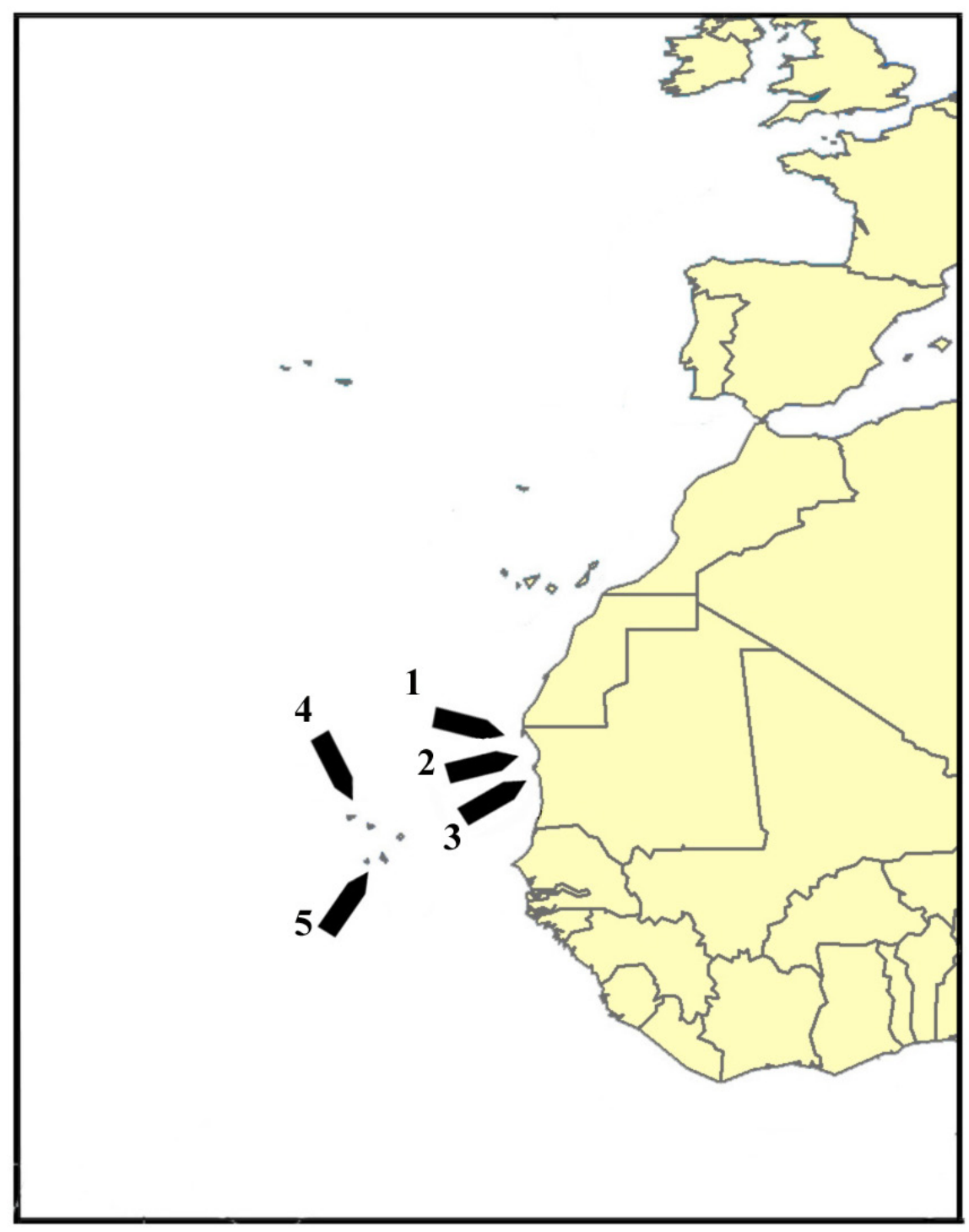

Fig. 1. Map of Northwest Africa, showing the approximate localities from which the sponges were collected. 1. Mauritania, Cap Blanc. 2. Mauritania, Banc d'Arguin. 3. Mauritania, SW of Cap Timiris 4. Cape Verde Islands, São Vicente 5. Cape Verde Islands, Ilheu de Cima. 


\title{
Material and methods
}

Specimens were collected by trawls, dredges and grabs during the CANCAP 7 Expedition (August 1986, Cape Verde Islands, on board of HNLMS Tydemann) and Mauritania II Expedition (June 1988, on board of RV Tyro) (Fig. 1). All material was provisionally identified on board and subsequently preserved in $96 \%$ ethanol. Until recently, the material was incorporated in the collections of the Zoological Museum of the University of Amsterdam (ZMA), but at present it is housed in the Netherlands Centre for Biodiversity Naturalis at Leiden. The acronym ZMA is maintained as part of the registration numbers of all specimens mentioned. Collection data are provided with each treatment of the species below. To study the skeletal structure, thick sections were made by hand, air-dried on a hotplate, and mounted in Canada Balsam. Stacked (automontage) light microscopy images of these thick sections were made using a Leica DM5500 microscope. For measurements of the spicules and SEM examination dissoluted spicule suspensions were made with concentrated $\mathrm{NaOHCl}$, washed five times in distilled water and mounted on light microscopic slides and SEM stubs. Spicule measurements (minimum-mean-maximum) are based on 25 spicules of each category or type for each individual specimen. SEM photos of spicules were combined on a black background, aligned, and cleaned using Photoshop CS3 licensed to R.W.M. Van Soest.

\section{Results}

\author{
Phylum Porifera Grant, 1836 \\ Class Demospongiae Sollas, 1885 \\ Order Poecilosclerida Topsent, 1928 \\ Suborder Mycalina Hajdu, Van Soest \& Hooper, 1994 \\ Family Esperiopsidae Hentschel, 1923
}

\section{Remarks}

Below we assign West African species to esperiopsid genera in accordance with the account of the family by Van Soest \& Hajdu (2002b). Species with only a single chela size, and no other microscleres are assigned to Amphilectus. The species with sigmas included is assigned to Esperiopsis. Species without microscleres are assigned to Ulosa.

Genus Amphilectus Vosmaer, 1880

\section{Remarks}

Amphilectus differs from the closely related genus Esperiopsis in the lack of additional microscleres and the generally smaller size of the styles. The difference in the latter character was fixed on $400 \mu \mathrm{m}$ (Van Soest \& Hajdu 2002b), but this is to be interpreted loosely, as several species in both genera possess styles with lengths just under or above $400 \mu \mathrm{m}$. The genus to date contains 17 species (Van Soest et al. 2012), predominantly from temperate and cold water, evenly spread over the North Atlantic (five species), North Pacific (three species), South Atlantic (six species), and South Pacific waters (three species). The Atlantic species are summarized in Table 1.

\section{Amphilectus utriculus sp. nov.}

Figs 2-3

\section{Etymology}

utriculus $(\mathrm{L})=$. small water bag, referring to the hollow, flattened shape. 
Table 1. Summary of characters of Atlantic sponges belonging to genera Amphilectus Vosmaer, 1880 and Esperiopsis Carter, 1882.

\begin{tabular}{|c|c|c|c|c|c|c|c|c|c|c|c|c|c|c|c|c|c|c|c|c|c|c|c|}
\hline 흥 & $\begin{array}{l}\overrightarrow{\widehat{C}} \\
5\end{array}$ & $\begin{array}{l}\text { 品 } \\
\text { 丵 }\end{array}$ & J & $r$ & 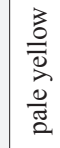 & 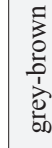 & 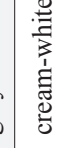 & ग्र & & مإِ & 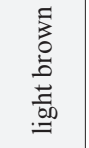 & $\frac{\mathscr{g}}{3}$ & 总 & 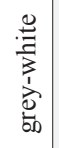 & r. & 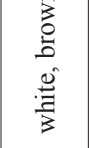 & 胥 & 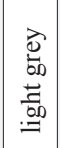 & 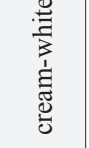 & $\begin{array}{l}\overrightarrow{\grave{b}} \\
\text { b. }\end{array}$ & 仓্ & 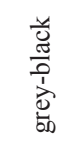 & $\stackrel{3}{\stackrel{3}{0}}$ \\
\hline$\overline{\bar{z}}$ & 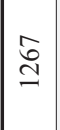 & $\frac{\infty}{0}$ & if & సี & $\begin{array}{l}\text { ֻे } \\
\text { ò }\end{array}$ & \& & స్లి & $\frac{\infty}{\frac{1}{2}}$ & & 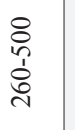 & 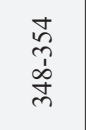 & 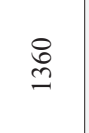 & 号 & $\&$ & 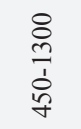 & $\begin{array}{l}\hat{a} \\
\hat{\sigma}\end{array}$ & 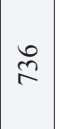 & $\infty$ & in & 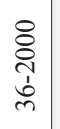 & $\frac{8}{\frac{0}{1}}$ & $\vec{m}$ & $\stackrel{2}{\frac{1}{n}}$ \\
\hline ๘ँ & $\stackrel{\overbrace{}}{\sharp}$ & $\stackrel{\mathscr{J}}{\sharp}$ & $\stackrel{\overbrace =}{\sharp}$ & 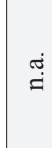 & 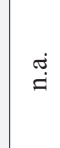 & $\stackrel{\overbrace{}}{\sharp}$ & 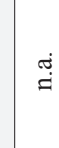 & $\stackrel{\overbrace{}}{=}$ & & 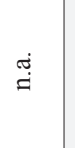 & ఝં & $\begin{array}{l}\stackrel{\bar{\Xi}}{\Xi} \\
\stackrel{\varrho}{O}\end{array}$ & 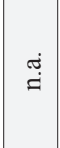 & 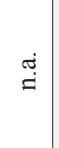 & 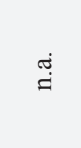 & $\cong$ & 㟛 & 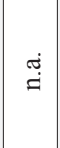 & $\stackrel{\leftrightarrow}{=}$ & 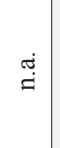 & $\stackrel{\overbrace{}]}{\sharp}$ & $\stackrel{\oplus}{\dot{\pi}}$ & 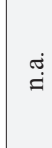 \\
\hline 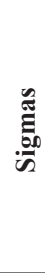 & 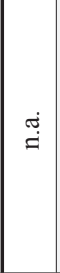 & 迅 & ঙ્円 & $\stackrel{\text { }}{g}$ & 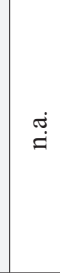 & ఝ્g & 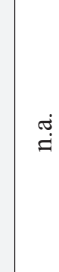 & 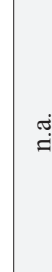 & & 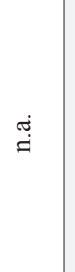 & ఝ્ & 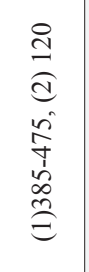 & 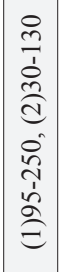 & 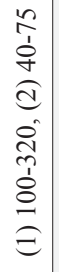 & 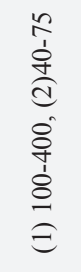 & $\begin{array}{l}\stackrel{n}{a} \\
\stackrel{d}{0} \\
\stackrel{n}{\Xi}\end{array}$ & 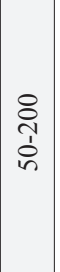 & $\stackrel{\Xi}{\sharp}$ & 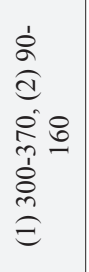 & \begin{tabular}{l}
8 \\
\multirow{1}{1}{} \\
0 \\
0
\end{tabular} & 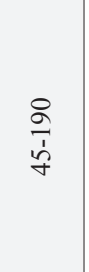 & 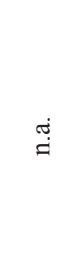 & 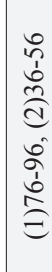 \\
\hline$\frac{\pi}{\varrho}$ & 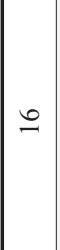 & $\begin{array}{l}\text { Tे } \\
\stackrel{I}{ \pm}\end{array}$ & $\frac{\tilde{I}}{\stackrel{d}{N}}$ & 角 & $\begin{array}{l}\text { ત̂. } \\
\text { ñ. }\end{array}$ & $\frac{n}{m}$ & $\begin{array}{l}\stackrel{\sim}{N} \\
\stackrel{N}{N}\end{array}$ & 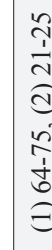 & & $\begin{array}{l}\text { Do } \\
\text { in }\end{array}$ & 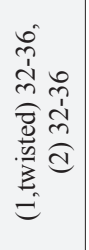 & 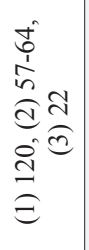 & 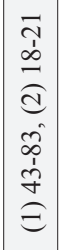 & 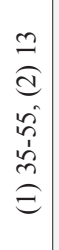 & 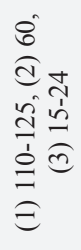 & in & $\mid \begin{array}{l}0 \\
\stackrel{2}{d} \\
\dot{8} \\
\stackrel{n}{\Xi}\end{array}$ & 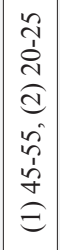 & 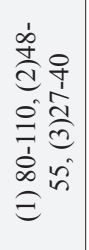 & 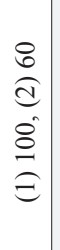 & 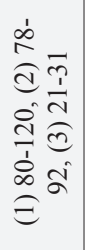 & 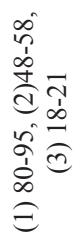 & 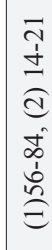 \\
\hline 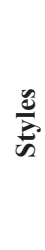 & $\mid \begin{array}{l}0 \\
x \\
x \\
o \\
n \\
n\end{array}$ & 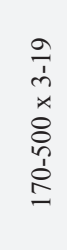 & 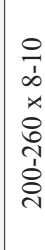 & 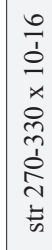 & 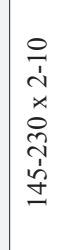 & 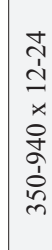 & 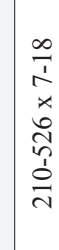 & 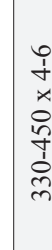 & & 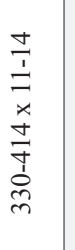 & 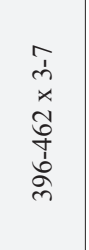 & $\begin{array}{l}0 \\
x \\
x \\
0 \\
n \\
o \\
o \\
0\end{array}$ & 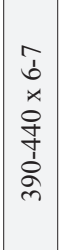 & 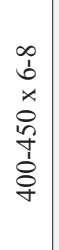 & 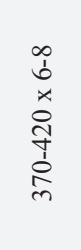 & $\begin{array}{l}m \\
\text { r. } \\
x \\
\infty \\
\infty \\
\infty \\
\hat{1} \\
\infty \\
\infty\end{array}$ & $\begin{array}{l}0 \\
x \\
x \\
b \\
y \\
y\end{array}$ & 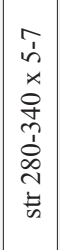 & 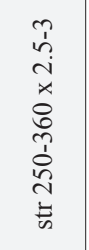 & $\begin{array}{l}r \\
\dot{f} \\
x \\
o \\
\dot{n}\end{array}$ & 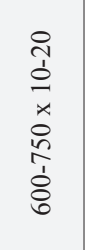 & $\begin{array}{l}\infty \\
i \\
\dot{x} \\
\tilde{n} \\
\tilde{y} \\
\dot{w} \\
\infty \\
\infty\end{array}$ & 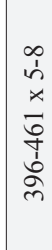 \\
\hline 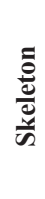 & 节 & 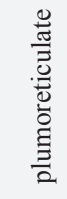 & $\frac{\frac{0}{\pi}}{\frac{\pi}{5}}$ & 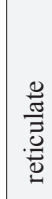 & 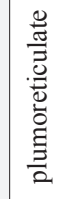 & 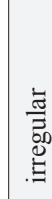 & | & $\begin{array}{l}\frac{0}{n} \\
\frac{\pi}{3} \\
\frac{3}{\tilde{z}}\end{array}$ & & 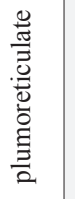 & 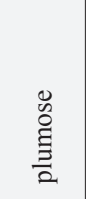 & 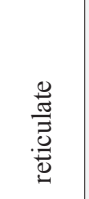 & 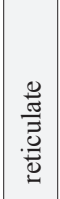 & 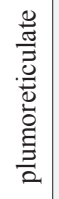 & 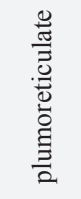 & 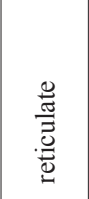 & 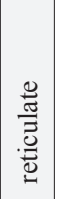 & 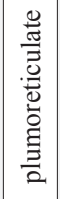 & 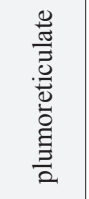 & 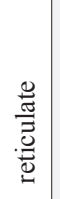 & 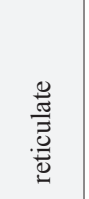 & 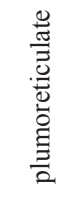 & $\begin{array}{l}\mathscr{0} \\
\ddot{\circ} \\
\text { 咅 }\end{array}$ \\
\hline$\cong$ & 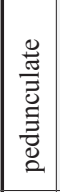 & 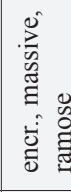 & 崽 & $\begin{array}{l}\ddot{\circ} \\
\stackrel{8}{0}\end{array}$ & 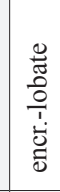 & 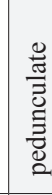 & 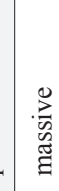 & 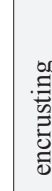 & & 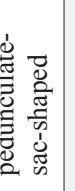 & 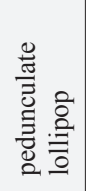 & 竭 & 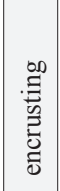 & 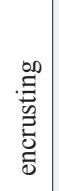 & 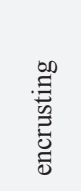 & 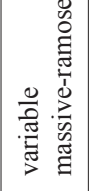 & 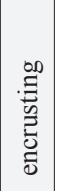 & 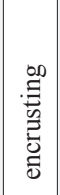 & 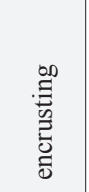 & 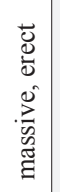 & 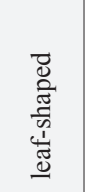 & 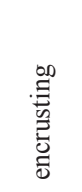 & 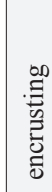 \\
\hline 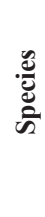 & 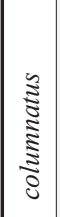 & 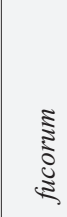 & 气 & $\Xi$ & 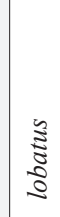 & 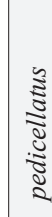 & 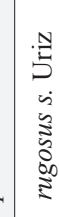 & 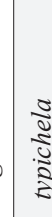 & & 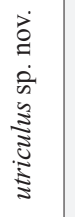 & 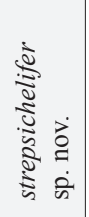 & $\frac{\tilde{0}}{\tilde{0}}$ & 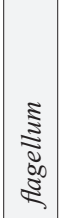 & 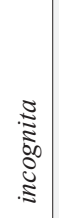 & 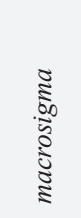 & 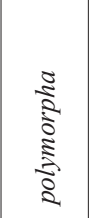 & 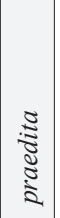 & 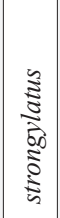 & 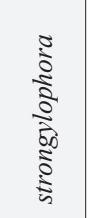 & 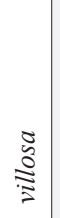 & 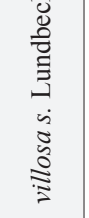 & & 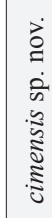 \\
\hline 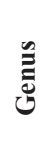 & 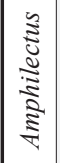 & 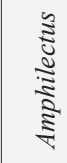 & $\stackrel{\Xi}{\Xi}$ & 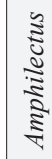 & 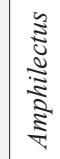 & 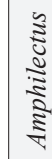 & 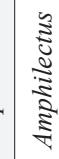 & 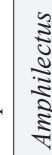 & & 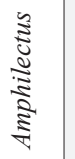 & 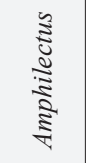 & 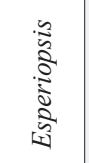 & 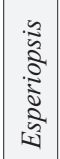 & 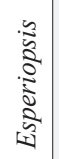 & 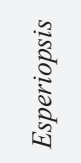 & 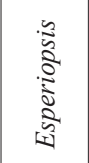 & 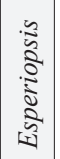 & 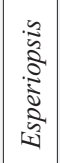 & 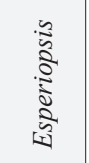 & 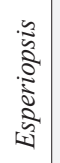 & 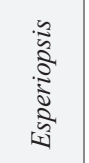 & 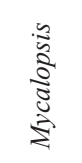 & 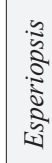 \\
\hline
\end{tabular}




\section{Material examined}

\section{Holotype}

ZMA Por. 22592, Mauritania, SW of Cap Timiris, depth 260-280 m, muddy bottom, coll. R.W.M. Van Soest \& J.J. Vermeulen, Mauritania II Expedition stat. 39/01, $18.8^{\circ} \mathrm{N} 16.7167^{\circ} \mathrm{W}, 3.5 \mathrm{~m}$ Agassiz trawl, 10 Jun. 1988.

\section{Paratypes}

ZMA Por. 06636, 53 specimens, Mauritania, SW of Cap Timiris, depth 260-280 m, muddy bottom, coll. R.W.M. Van Soest \& J.J. Vermeulen, Mauritania II Expedition stat. 39/01, $18.8^{\circ} \mathrm{N} 16.7167^{\circ} \mathrm{W}, 3.5 \mathrm{~m}$ Agassiz trawl, 10 Jun. 1988. ZMA Por. 06668, Mauritania, SW of Cap Timiris, depth $500 \mathrm{~m}$, fossil coral debris, coll. R.W.M. Van Soest \& J.J. Vermeulen, Mauritania II Expedition stat. $40 / 20,18.85^{\circ} \mathrm{N}$ $16^{\circ} 8833^{\circ} \mathrm{W}, 3.5 \mathrm{~m}$ Agassiz trawl, 10 Jun. 1988.
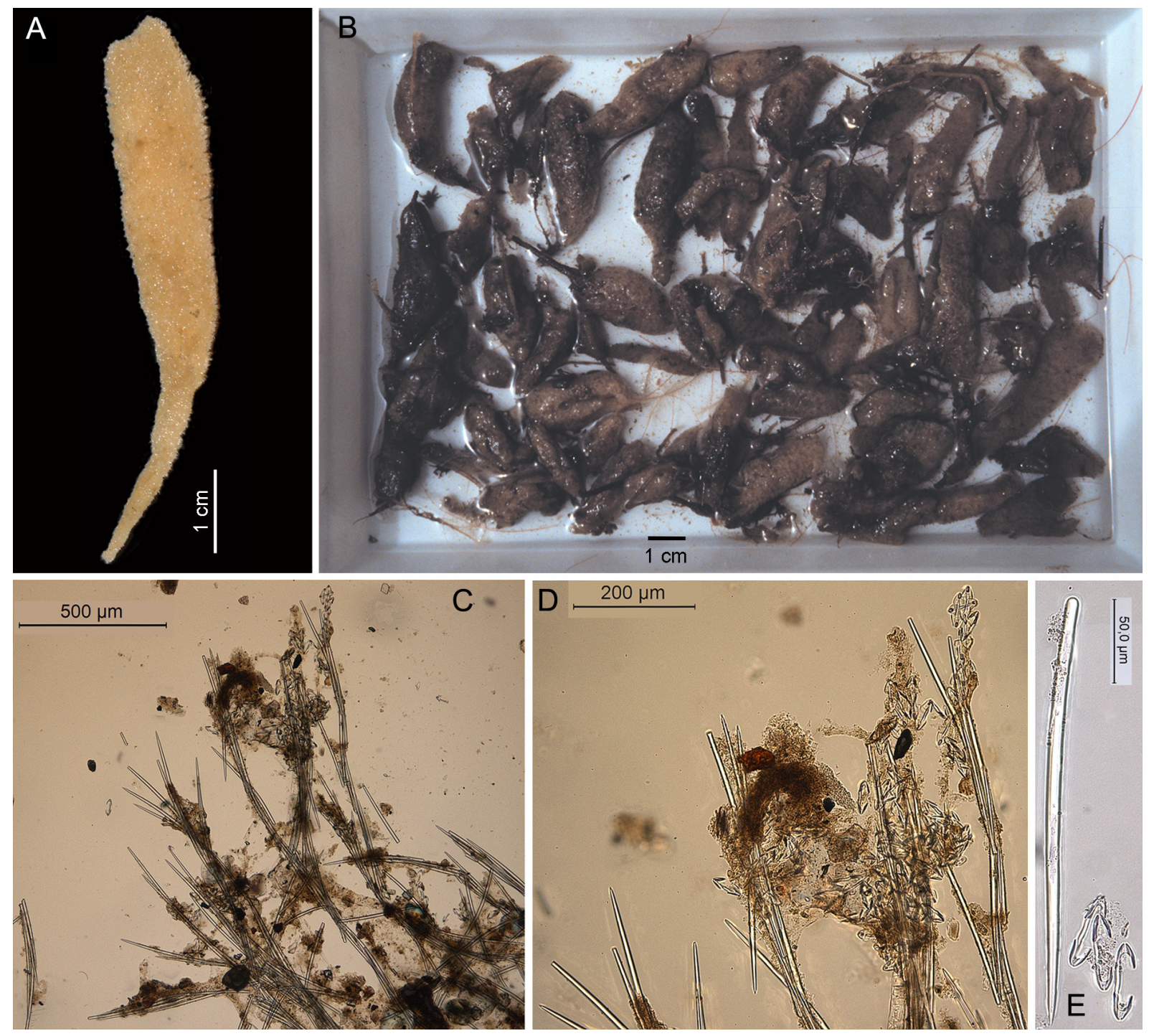

Fig. 2. Amphilectus utriculus sp. nov. A,C-E. Holotype ZMA Por. 22592. B. Paratypes ZMA Por. 06636. C. Cross section showing skeletal structure. D. Peripheral skeleton showing protruding spicule tracts and clusters of microscleres. E. Spicules in light microscopy. 


\section{Additional specimen}

ZMA Por. 06627, Mauritania, SW of Cap Timiris, depth 200 m, muddy sand, coll. R.W.M. Van Soest \&

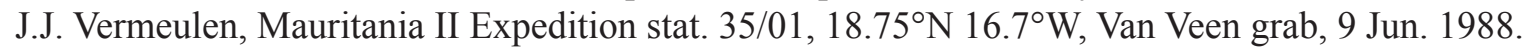

\section{Description}

The holotype (Fig. 2A) and most other specimens (Fig. 2B) are short-stalked, with main body laterally flattened, isodiametrical or either tapering inward near the upper end or flaring, with smaller or wider opening. Main body hollow, like a purse. Colour reddish or greyish brown, both alive and in alcohol. Surface irregular, shaggy. Consistency very soft, limp, easily damaged. Stalk rounded, but grading into the main body by widening, surface slightly smoother. Size $6 \mathrm{~cm}$ high (holotype), up to $8 \mathrm{~cm}$ high (paratypes), $0.8 \mathrm{~cm}$ (holotype), up to $1 \mathrm{~cm}$ (paratypes) in widest expansion, proportion of stalk and main body approximately $1: 3$.

SkeLETon. There is no special ectosomal skeleton, skeletal bundles are simply protruding beyond the surface. Choanosomal skeleton consists of polyspicular bundles (Fig. 2C), with 3-6 spicules in cross section, connected regularly by 1-2 spicules at right angles. Near the surface the bundles fan out to form loose brushes. Microscleres are concentrated lining the bundles and crowding the brushed endings at the surface (Fig. 2D). In the interior, microscleres are scattered and distinctly less numerous than at the surface.

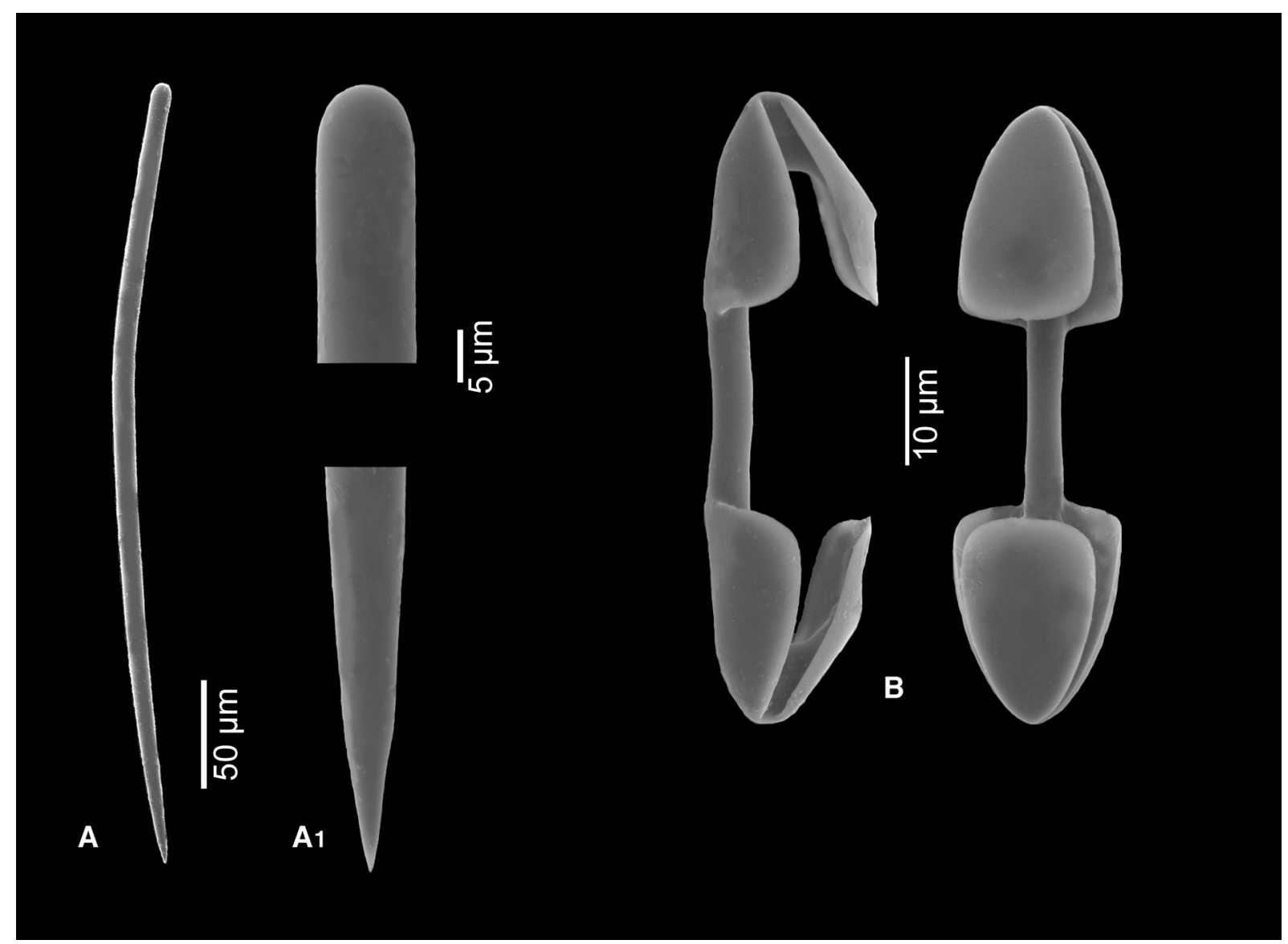

Fig. 3. Amphilectus utriculus sp. nov., Holotype ZMA Por. 22592, SEM images of spicules. A. Styles. $\mathbf{A}_{1}$. Detail of the apices. B. Palmate isochelae in side and ventral view. 
Spicules. (Figs 2E, 3) Styles, palmate isochelae.

StYLes. (Fig. 3A, $\mathrm{A}_{1}$ ) Of the mycalostyle-type, slightly constricted near the rounded end, straight, or more commonly slightly curved, 330-371.2-414 x 11-12.7-14 $\mu \mathrm{m}$.

Palmate isochelae. (Fig. 3B) 'Normal'-shaped, but with the frontal alae appearing somewhat angular in side-view, with the shaft slightly incurved, all closely similar in size, 52-59.6-66 $\mu \mathrm{m}$.

\section{Distribution and ecology}

Mauritania, S of Banc d'Arguin (Fig. 1, loc. 3), muddy bottom at 200-500 m depth.

\section{Remarks}

Assignment to the genus Amphilectus is based on the possession of a single microsclere type, although technically speaking the size of the styles exceeds the $400 \mu \mathrm{m}$ upper size given as an additional character of Amphilectus. North Atlantic Amphilectus species with pedunculate shape were reviewed from literature descriptions (see also Table 1). A. columnatus (Topsent, 1890) (as Esperiopsis) from deepsea waters around the Azores has much longer styles $(750 \mu \mathrm{m})$ and much smaller isochelae $(16 \mu \mathrm{m})$. A. pedicellatus (Lundbeck, 1905) (as Esperiopsis) from West Greenland likewise has longer styles (up to $940 \mu \mathrm{m})$ and very small chelae $(13-15 \mu \mathrm{m})$. Further species are not only different in shape, but also show spicular differences: A. fucorum (Esper, 1794) (as Spongia) is massively-encrusting to digitateramose and has smaller spicules overall (see also below). A. lobatus (Esper, 1794) (as Spongia) is lobate and likewise has smaller spicules, and the isochelae are verging toward an anisochelate condition. Both A. fucorum and A. lobatus are essentially shallow-water species. A. typichela (Lundbeck, 1905) (as Esperiopsis) is encrusting and has two distinct size classes of isochelae.

Southward along the African coasts, in Namibian and South African waters, three further species have been recorded: A. rugosus sensu Uriz (1988) [as Esperiopsis, probably not conspecific with the Chilean species A. rugosus (Thiele, 1905)] is a whitish massive sponge with much smaller isochelae. A. lesliei (Uriz, 1988) (as Esperiopsis) is ramose and has strongyles as megascleres, distinctly smaller than the styles of the new species. A. informis (Stephens, 1915) is encrusting and has much smaller spicules.

ZMA Por. 06627 from Mauritania (stat. 35/01) is a small whitish crust showing the same skeletal structure and a spicule complement slightly smaller but essentially similar to the above described specimens: styles 302-329.5-353 x 6-9.1-12 $\mu \mathrm{m}$, palmate isochelae: 33-43.5-51 $\mu \mathrm{m}$. For the time being this is assumed to be an incipient individual of $A$. utriculus sp. nov., but the colour and the encrusting habit point to a possible separate species.

\section{Amphilectus strepsichelifer sp. nov.}

Fig. 4

\section{Etymology}

The name is a combination of strepsis $(\mathrm{L})=$. twisted, and chelifer $(\mathrm{L})=$. bearing chelae, reflecting the twisted condition of the chelae.

\section{Material examined}

\section{Holotype}

ZMA Por. 07564, Cape Verde Islands, W of São Vicente, Canal de São Vicente, depth 348-354 m, coll. R.W.M. Van Soest, CANCAP 7 Expedition stat. $172 / 03,16.8833^{\circ} \mathrm{N} 25.1167^{\circ} \mathrm{W}$, rectangular dredge, 7 Sep. 1986. 


\section{Description}

Pedunculate sponge (Fig. 4A), with long thin smooth stalk and abruptly attached small ovate main body. Main body flattened, but solid (not hollow). Upper surface somewhat rectangular, caused by preparation damage. Surface irregular, shaggy. Colour light brown alive, grey in alcohol. Size of main body $12 \mathrm{x}$ $6 \mathrm{~mm}$, stalk $35 \mathrm{~mm}$ long, $1.2 \mathrm{~mm}$ thick.

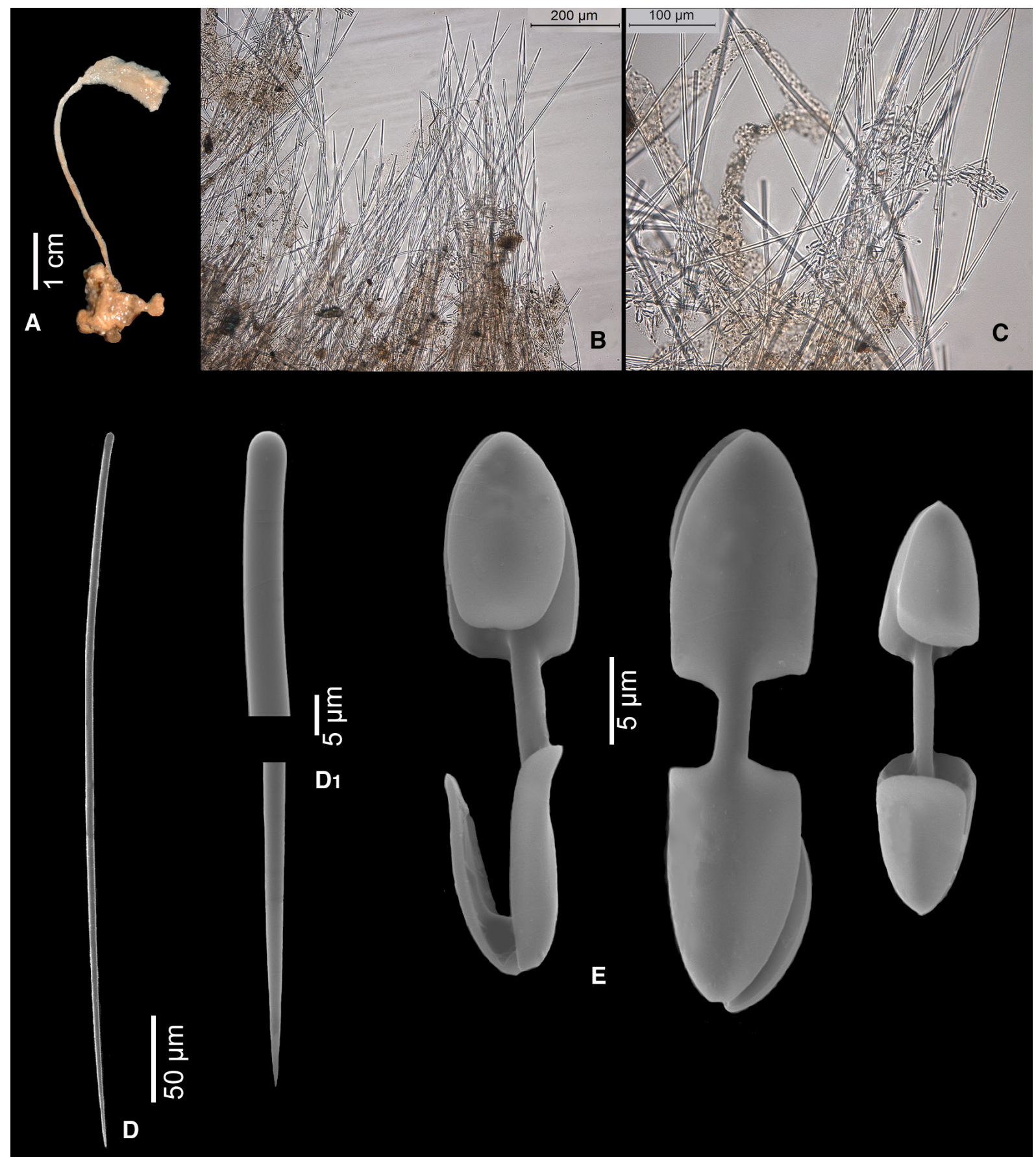

Fig. 4. Amphilectus strepsichelifer sp. nov., Holotype ZMA Por. 07564. A. Habit. B-C. Cross section of skeleton in light microscopy. B. Peripheral skeleton. C. Detail of peripheral tracts and clustered microscleres. D-E. SEM images of spicules. D. Style. D $_{\mathbf{1}}$. Details of apices. E. Palmate isochelae, in various stages of torsion. 
Skeleton. Of main body plumose, rather than plumoreticulate, with diffuse spicule bundles directed at right angles to the surface (Fig. 4B), where they form loose brushes. Connecting spicules few and arranged irregularly. Chelae in loose groups in a subectosomal layer at the base of the surface brushes (Fig. 4C). Few chelae in the interior.

SpICULES. (Fig. 4D-E) Styles, palmate isochelae.

StYLes. (Fig. 4D, $\mathrm{D}_{1}$ ) Thin, slightly curved, 396-430.5-462 x 3-4.6-7 $\mu \mathrm{m}$.

Palmate isochelae. (Fig. 4E) Predominantly with 'twisted' shaft, causing the alae of opposite ends to face different angles, a minority of the chelae appearing 'normal' but these are of the same size as the twisted ones, and upon closer examination appear to be slightly twisted as well, 32-33.7-36 $\mu \mathrm{m}$.

\section{Distribution and ecology}

Known only from the type locality between the islands of São Vicente and Santo Antão, Cape Verde Islands (Fig. 1, loc. 4), hard bottom, at depths below $300 \mathrm{~m}$.

\section{Remarks}

The species is assigned to Amphilectus on the same basis as A. utriculus sp. nov. The species stands out among stalked Amphilectus species (see above in the remarks on A. utriculus sp. nov.) by the peculiar twisted condition of the palmate isochelae. Additionally the thin stalk carrying the main body without a clear intermediate zone is characteristic and not found in the other North Atlantic Amphilectus species.

Amphilectus cf. fucorum (Esper, 1794)

Fig. 5

For synonymy, see Van Soest \& Hajdu 2002b: 657.

\section{Material examined}

ZMA Por. 06775, Mauritania, off Banc d'Arguin, depth 100 m, bottom muddy sand, coll. R.W.M. Van

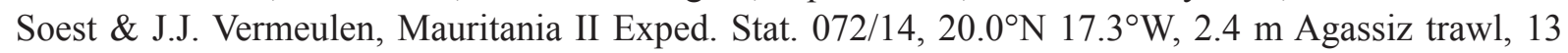
Jun. 1988. ZMA Por. 06796, Mauritania, off Banc d'Arguin, depth 48-52 m, bottom muddy sand with some calcareous gravel, coll. R.W.M. Van Soest \& J.J. Vermeulen, Mauritania II Exped. Stat. 082/19, $19.9833^{\circ} \mathrm{N} 17.5^{\circ} \mathrm{W}, 3.5 \mathrm{~m}$ Agassiz trawl, 14 Jun. 1988. ZMA Por. 06843, Mauritania, off Banc d'Arguin, depth 95-100 m, bottom muddy sand with shells, coll. R.W.M. Van Soest \& J.J. Vermeulen, Mauritania II Exped. Stat. 130/09, 20.4167 N 17.6667 W, 3.5 m Agassiz trawl, 20 Jun. 1988.

\section{Description}

The material consists of several fragments of encrusting to irregularly ramose sponges (Fig. 5A). Consistency, soft irregular surface, colour brownish alive and beige to whitish in alcohol. Size of individual fragments $2-3 \mathrm{~cm}$.

SKELETON. Irregularly plumoreticulate (Fig. 5B), with loosely defined spicule bundles connected by individual spicules, general aspect rather confused. Spicules barely protruding beyond the surface. Chelae clustered and singly occurring throughout the interior.

Spicules. Styles, palmate isochelae.

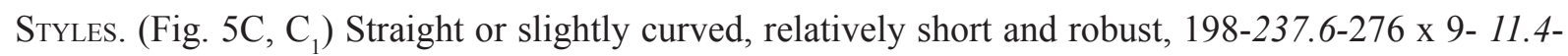
$14 \mu \mathrm{m}$. 
Palmate isochelae. (Fig. 5D) Of 'normal' shape, but like in A. utriculus sp. nov., the shaft is slightly incurved. 19-23.6-27 $\mu \mathrm{m}$.

\section{Distribution and ecology}

Mauritania, off Banc d'Arguin (Fig. 1, loc. 2), on muddy bottom below $50 \mathrm{~m}$. Elsewhere, if identification is correct, along most of the coasts of Europe, including the Western Mediterranean. This is the southernmost record of the species if Southern Ocean records (see below) are considered not conspecific.

\section{Remarks}

By assigning these specimens to A. fucorum, the range of this species, which was already huge, is further extended along the East Atlantic coasts. The species is common and distinctly orange-coloured in shallow water habitats of the British Isles and the W coast of France, but according to Van Soest et al.

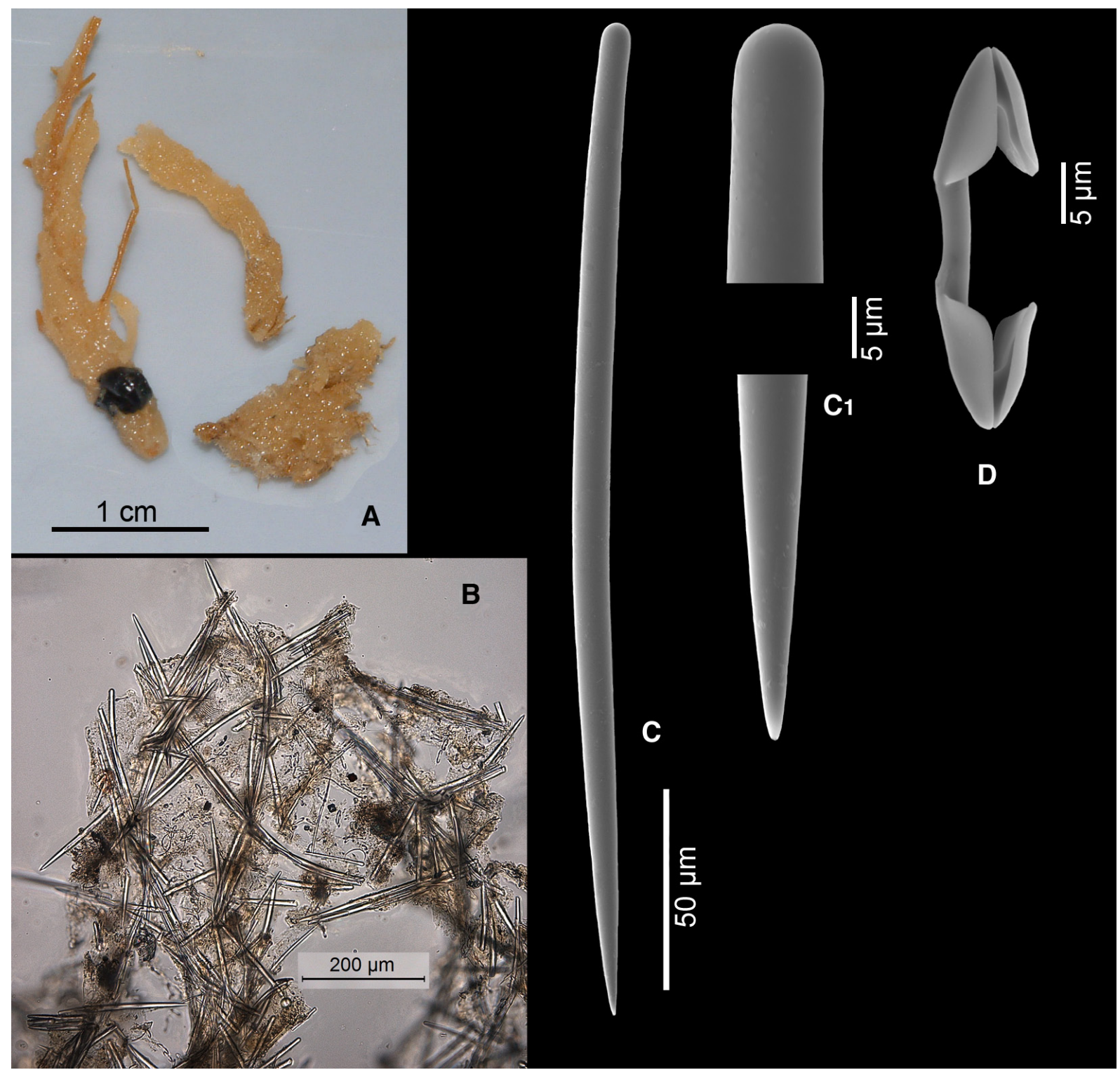

Fig. 5. Amphilectus fucorum (Esper, 1794), ZMA Por. 06775. A. Habit (fragments). B. Cross section of skeletal reticulation. C-D. SEM images of spicules. C. Style. C. Details of apices. D. Palmate isochela. 
(2000) deep-water specimens may loose their colour, and such specimens may be found down to $100 \mathrm{~m}$. Skeleton and spicule characteristics of the present material fall within the recorded variation, although usually styles elsewhere are thinner than those of the Mauritanian specimens. Genetic comparisons may show diversity over the range of this species and such studies are needed to decide the specific identity of the Mauritanian populations.

From the other Amphilectus species from the area described above, A. cf. fucorum differs in habit and chelae (A. utriculus sp. nov. has chelae twice the size, A. strepsichelifer sp. nov. has chelae with a twisted shaft).

Amphilectus informis (Stephens, 1915) from the Atlantic coast of South Africa appears to be similar based on published data. According to the original description its chelae have an incurved shaft but apparently the frontal alae of the chelae are characteristic showing a 'tubercle in front view'. However, the illustration of this feature is indistinct. Samaai \& Gibbons (2005) described the species also, but their illustrations do not clarify these alleged differences.

Burton $(1932,1940)$ recorded A. fucorum from Tristan da Cunha, the Falkland Islands, South Georgia, and off the coast of Argentina. Thiele (1905) recorded it from Chile. Goodwin et al. (2011) described two new Amphilectus species from the Falkland Islands, which appear to cover the Burton and Thiele records. Bergquist \& Fromont (1988) recorded the European species Esperiopsis normani (Bowerbank, 1866) and Esperiopsis edwardii (Bowerbank, 1866) from New Zealand waters, but both are now considered junior synonyms of Amphilectus fucorum. This is not to say that A. fucorum occurs in New Zealand, but merely that these records should be compared critically.

\section{Remarks}

Genus Esperiopsis Carter, 1882

Currently, there are 33 species assigned to this genus (Van Soest et al. 2012). Possibly, some of the species may need to be transferred to other genera because of the interpretation of the morphological structure of the chelae (e.g. E. flava Lévi, 1993 and similar species with deformed chelae, which cannot be identified with certainty as palmate), others would have to be returned to Amphilectus if the style size difference would be no longer a valid discriminatory character of the two genera. Future studies using additional datasets (such as molecular sequences) are needed to clarify the status of Esperiopsis.

Esperiopsis cimensis sp. nov.

Fig. 6

\section{Etymology}

Named after the type locality, Ilheu de Cima.

\section{Material examined}

\section{Holotype}

ZMA Por. 07282, Cape Verde Islands, SW of Ilheu Rombos, SE of Ilheu de Cima, depth 165 m, hard bottom with yellow calcareous sand, coll. R.W.M. Van Soest, CANCAP 7 Expedition stat. 030/05, $14.95^{\circ} \mathrm{N} 24.65^{\circ} \mathrm{W}$, Van Veen grab, 23 Aug. 1986.

\section{Description}

Thinly encrusting on a large volcanic stone (Fig. 6A, arrow), alongside a specimen of Aplysilla Schulze, 1878. Size of crust $3.5 \times 2 \mathrm{~cm}$, thickness $1-2 \mathrm{~mm}$. Colour: yellow alive, beige in alcohol. Surface optically smooth. Consistency soft. 


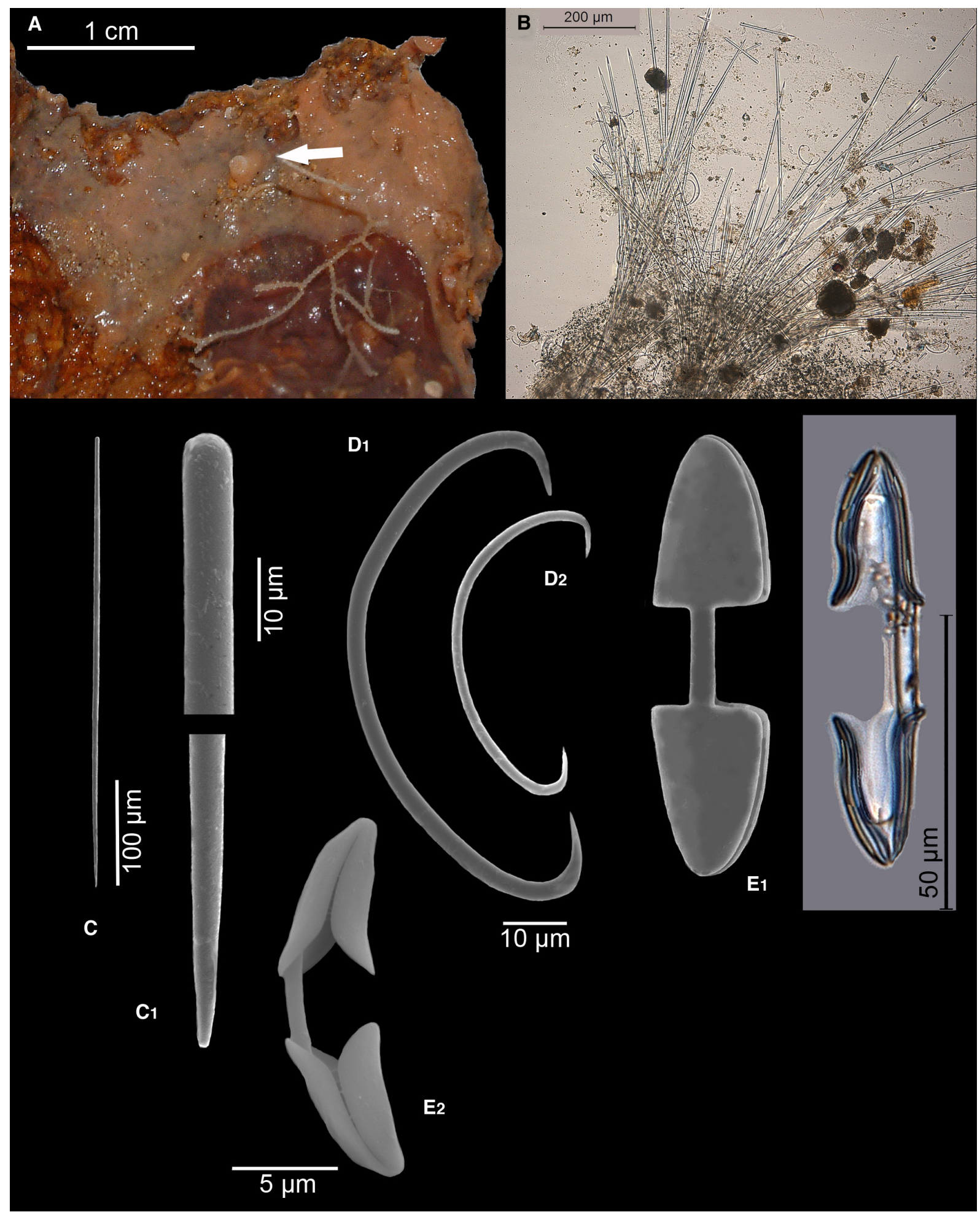

Fig. 6. Esperiopsis cimensis sp. nov., Holotype ZMA Por. 07282. A. Habit encrusting a volcanic rock (indicated by arrow). B. Cross section of peripheral skeleton showing plumose spicule brushes. C-E. SEM and light microscopy images of spicules. D. Style. $\mathbf{D}_{\mathbf{1}}$. Detail of apices. C. Sigmas. $\mathbf{C}_{\mathbf{1}}$. Large sigma. $\mathbf{C}_{2}$. Small sigma. E. Palmate isochelae. $\mathbf{E}_{\mathbf{1}}$. Left: large chela in dorsal view; right: large chela in side view. $\mathbf{E}_{2}$. Small chela. 
Skeleton. (Fig. 6B) Plumose, with strongly developed spicule bundles traversing the sponge at right angles to the surface, where they fan out. Few if any connecting spicules. No special ectosomal skeleton. There is a 'groundmass' of microscleres crowding the space between the spicule bundles and adhering to it, mostly consisting of sigmas and small isochelae. Scattered larger chelae, rather rare, mostly occurring at the surface.

Spicules. (Fig. 6C-E) Styles, sigmas, palmate isochelae.

STYLEs. (Fig. 6C, $\mathrm{C}_{1}$ ) Long and thin, straight or slightly curved, 396-431.4-461 x 5-6.4-8 $\mu \mathrm{m}$.

Sigmas. (Fig. 6D) In two distinct non-overlapping size categories, both thin and strongly curved: (I) 76-

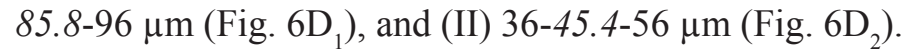

Palmate isochelae. (Fig. 6E) In two distinct, non-overlapping size categories, the larger (I) 'normal' in

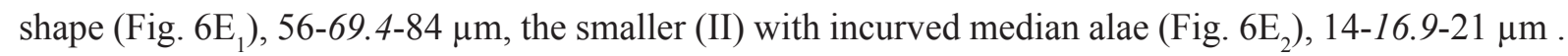

\section{Distribution and ecology}

Only known from the type locality S of Ilheu de Cima, Cape Verde Islands (Fig. 1, loc. 5), on volcanic bottom with yellow sand, at $165 \mathrm{~m}$ depth.

\section{Remarks}

The new species appears to be a member of a group of morphologically similar North Atlantic deep-water species (see Table 1), sharing the encrusting habit, the size and shape of megascleres and two categories of both sigmas and chelae. Probably closest is Esperiopsis flagellum Lundbeck, 1905 from off SE Iceland. Clear differences exist in the shape of one of the sigma categories, as these of E. flagellum are 'flagellate' (excessively incurved). The sigmas are also larger in size, up to $250 \mu \mathrm{m}$ in E. flagellum, whereas they are up to $400 \mu \mathrm{m}$ in the similar species E. macrosigma Stephens, 1916 from waters west of Ireland. E. praedita Topsent, 1890 from the Azores is also close, but the larger chelae category is smaller than in our new species, and the sigmas can be as large as $200 \mu \mathrm{m}$. A less similar sponge is E. decora Topsent, 1904 from the Azores, sharing the overall spiculation, but having even larger (flagellate) sigmas, and in addition possessing trichodragmas and three instead of two size categories of chelae. Other Esperiopsis species described from the North Atlantic appear more distant: E. incognita Stephens, 1916 from Irish waters has smaller chelae and larger sigmas. E. strongylata (Alander, 1942) from the Skagerrak and E. strongylophora Vacelet, 1969 from the Western Mediterranean have strongylote megascleres instead of styles. The two remaining, more elaborately shaped, North East Atlantic Esperiopsis species also show differences in spicule sizes and categories: variably massive or ramose E. polymorpha Topsent, 1890 from the Azores has smaller chelae in a single category and smaller sigmas. Massive-erect or leafshaped E. villosa (Carter, 1874) has the size of the largest sigmas and chelae clearly in excess of those of our new species.

Esperiopsis schmidti Arnesen, 1903 from Norwegian waters and Esperiopsis glomeris Topsent, 1904 have been assigned to the myxilline genus Echinostylinos Topsent, 1927 by Van Soest \& Hajdu (2002a) on account of their possession of tridentate isochelae. Esperiopsis typichela Lundbeck, 1905 and Esperiopsis pedicellata Lundbeck, 1905 have been transferred to Amphilectus by Van Soest et al. (2012) as they do not possess sigmas. Esperiopsis lesliei Uriz, 1988 and Esperiopsis rugosus sensu Uriz (1988) both from Namibia, likewise do not have sigmas and belong to Amphilectus. 
Genus Ulosa de Laubenfels, 1936

Remarks

The genus Ulosa is not well-established, with approximately 15 disjunctly occurring species (South Australia, North East Atlantic and Mediterranean, Indonesia, Central Pacific, and Brazil, see Van Soest et al. 2012). About half the number of species are only known from old dried types and/or are inadequately characterized. With the simple morphological features the genus remains to be established as a monophyletic group, and indeed as a member of the suborder Poecilosclerida, as the implied assumption of secondarily lost chelae lacks firm evidence. In fact, a recent study of $28 \mathrm{~S}$ gene sequences of a large group of demosponges including poecilosclerid, hadromerid and halichondrid sponges (Morrow et al. 2012) indicated that at least one species of the genus Ulosa could be a member of the family Halichondriidae Gray, 1867. Here we will retain the genus as a member of Esperiopsidae until sequences of the type species of Ulosa will be obtained.

\section{Ulosa stuposa (Esper, 1794)}

Fig. 7

For synonymy, see Van Soest 1987.

\section{Material examined}

ZMA Por. 06567, Mauritania, off Cap Blanc, depth 15 m, bottom muddy, coll. R.W.M. Van Soest \& J.J. Vermeulen, Mauritania II Exped. Stat. 006/01, 20.8167 $\mathrm{N} 17.0167^{\circ} \mathrm{W}, 1.2 \mathrm{~m}$ Agassiz trawl, 7 Jun. 1988. ZMA Por. 06705, Mauritania, SW of Cap Timiris, depth 12-18 m, bottom sand overlying limestone ridge, coll. R.W.M. Van Soest \& J.J. Vermeulen, Mauritania II Exped. Stat. 049/18, 19.0833 ${ }^{\circ} \mathrm{N} 16.4167^{\circ} \mathrm{W}$, rectangular dredge, 11 Jun. 1988. ZMA Por. 06714, Mauritania, SW of Cap Timiris, bottom muddy sand, depth 30 m, coll. R.W.M. Van Soest \& J.J. Vermeulen, Mauritania II Exped. Stat. 053/05, 19.0833 ${ }^{\circ} \mathrm{N}$ 16.4667 W, $2.4 \mathrm{~m}$ Agassiz trawl, 11 Jun. 1988. ZMA Por. 06766, Mauritania, off Banc d'Arguin, bottom muddy sand, depth 48-52 m, coll. R.W.M. Van Soest \& J.J. Vermeulen, Mauritania II Exped. Stat.

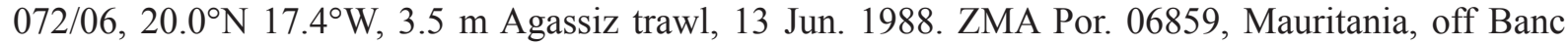
d'Arguin, bottom muddy sand, depth 50 m, coll. R.W.M. Van Soest \& J.J. Vermeulen, Mauritania II Exped. Stat. 137/01, $20.7167^{\circ} \mathrm{N} 17.35^{\circ} \mathrm{W}, 2.4 \mathrm{~m}$ Agassiz trawl, 21 Jun. 1988.

\section{Examined for comparison}

ZMA Por. 09932, Portugal, Algarve, E of Sagres, $37.0^{\circ} \mathrm{N} 8.9333^{\circ} \mathrm{W}$, growing on Cystoseira alga, coll. J.H. Stock, 14 Aug. 1976. ZMA Por. 16490, France, Bretagne, Roscoff, Le Taureau, depth 25 m, $48.6633^{\circ} \mathrm{N} 3.8833^{\circ} \mathrm{W}$, coll. M.J. de Kluijver, Jul. 1992.

Ulosa digitata sensu Burton 1956, slide made from a specimen in the collections of the Zoological Museum of Copenhagen collected by the Atlantide Expedition Stat. 147, $09.4333^{\circ} \mathrm{N} 14.9667^{\circ} \mathrm{W}$, depth $45 \mathrm{~m}$, bottom shells and forams, 14 Apr. 1946.

\section{Description}

Long branches (Fig. 7A) issuing from a basal mass (presumably buried in the sand), dividing sparingly, surface irregularly conulose. Length of branches up to $10 \mathrm{~cm}$, diameter $0.5-1.5 \mathrm{~cm}$. Consistency soft, easily damaged.

CoLour. Variously reported as yellow, light brown, brown, blue-green or greyish. 
Skeleton. (Fig. 7B) A rectangular reticulation of thick spicule tracts, cemented by only little spongin, which is usually not visible. Tracts divisible in slightly thicker longitudinals of 35-100 $\mu \mathrm{m}$ thickness with a core of up to 15 spicules, and thinner interconnecting tracts of 30-60 $\mu \mathrm{m}$ thickness with a core of 2-10 spicules. Meshes formed by the tracts of widely divergent sizes 200-750 $\mu \mathrm{m}$. Loose spicules are observed among the tracts, but these are absent or rare in many specimens, which appear to be 'macerated', possibly due to loss of tissue during alcohol preservation.

Spicules. (Fig. 7C, $\mathrm{C}_{1}$ ) Styles only (no oxeote modifications were observed), sharply pointed, tending to be thicker in the middle than at the rounded end ('fusiform'), occasionally faintly 'centrotylote', of rather variable size in the various specimens, but with limited variation within a specimen, 123-161.2$192 \times 4-7.6-11 \mu \mathrm{m}$.

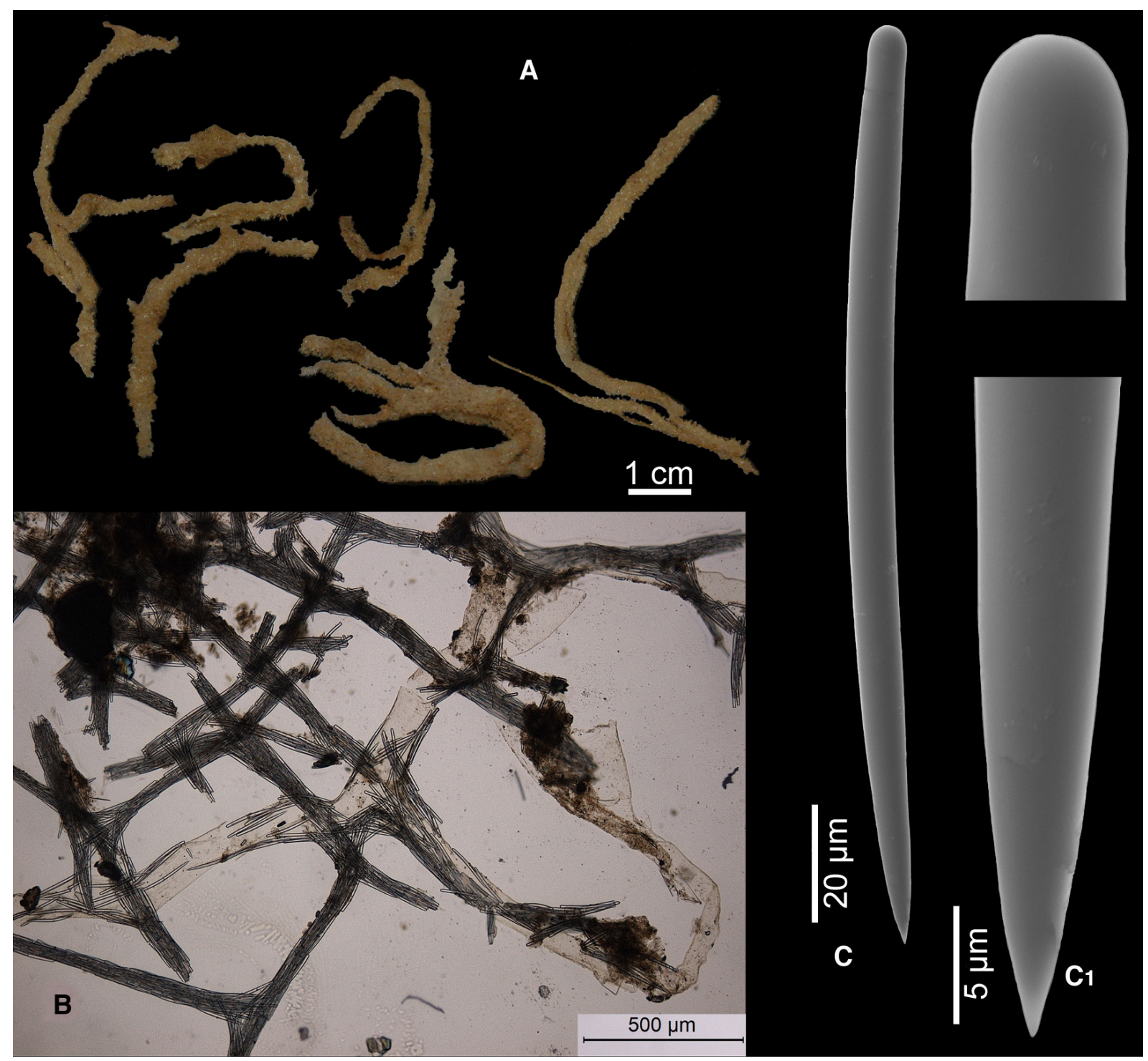

Fig. 7. Ulosa stuposa (Esper, 1794), ZMA Por. 06766. A. Fragmented habit. B. Cross section of skeleton. C. SEM image of style. $\mathbf{C}_{1}$. Detail of apices. 


\section{Distribution and ecology}

Mauritania, Cap Blanc, Banc d'Arguin and Cap Timiris, in sandy substrate at 12-52 m (Fig. 1, locs. 1-3). Elsewhere, widespread from the British Isles southward, Mediterranean, Lusitanian region, Atlantic islands; southernmost record Gulf of Guinea (see below).

\section{Remarks}

The West African material shows a range of growth forms including massively encrusting and branchingerect, in accordance with the variability elsewhere. Colours in Mauritanian specimens appear duller (mostly brownish) than in Western European material (cf. Van Soest et al. 2000) and spicule thickness on average appears greater than in northern specimens: in two specimens from Western France (ZMA Por. 16490) and Portugal (ZMA Por. 09932), examined for comparison, spicules measured 140-165 x 2-6 $\mu \mathrm{m}$. Nevertheless, there is overlap in these characters and conspecificity seems likely.

Burton (1956) reported this species (as $U$. digitata) from Conakry, Guinea $\left(9.4667^{\circ} \mathrm{N} 14.9667^{\circ} \mathrm{W}\right)$. It was described as forming a bush with branches $5-6 \mathrm{~cm}$ long and $0.5 \mathrm{~cm}$ thick. The skeleton is similar to Western European specimens and spicules conform as well (130-145 x 3-5 $\mu \mathrm{m})$. Apparently, the distribution of $U$. stuposa extends over much of the coastal waters of the Northeast Atlantic.

A littoral species from Faial, Azores, Ulosa jullieni (Topsent, 1892) (1892: 137, pl. I, fig. 12, as Stylinos), has similar skeleton with slightly smaller spicules $(120-130 \mu \mathrm{m})$, but differs from $U$. stuposa primarily in growth form and surface (thickly encrusting with smooth surface and slightly elevated oscules). It is also recorded here from the piles of the pier of Villa Baleira, Porto Santo, $33.0562^{\circ} \mathrm{N} 16.334^{\circ} \mathrm{W}$ (ZMA Por. 21503, coll. R.W.M. Van Soest, 30 June 1990).

Ulosa capblancensis sp. nov.

Fig. 8

\section{Etymology}

Named after the type locality, Cap Blanc.

\section{Material examined}

\section{Holotype}

ZMA Por. 09959, Mauritania, off Cap Blanc, depth 11-35 m, coll. F.P. Vermeulen, $20.7^{\circ} \mathrm{N} 17.1667^{\circ} \mathrm{W}$, 1906.

\section{Description}

Erect branches issue from a basal mass (Fig. 8A), which was presumable buried in the sandy substrate. Individual branches $8 \mathrm{~mm}$ diameter at the base tapering to blunt endings of 2 to $4 \mathrm{~mm}$ diameter. Individual branches up to $10 \mathrm{~cm}$ long. Basal mass (Fig. $8 \mathrm{~A}_{1}$ ) 3-6 cm thick, 4-5 cm in lateral expansion. Surface of basal mass and branches roughly conulose and porose, consistency rather firm, but soft to the touch. No apparent oscules. Possibly the specimen is somewhat macerated.

Colour. Brownish red in alcohol, live colour unknown.

Skeleton. (Fig. 8B) Forms a spongin-rich irregular reticulation with longitudinal meshes. Emphasis of the skeleton is on ascending fibres, which are cored by 2-9 spicules, whereas cross connections are predominantly cored by single spicules at widely differing angles, occasionally two or more spicules are in the connecting spongin-fibres but then these are not aligned. Meshes average $500 \mu \mathrm{m}$ in length, 
distance between longitudinal fibres is $200-400 \mu \mathrm{m}$, fibres have a thickness of 100-220 $\mu \mathrm{m}$ depending not only on the amount of coring but also on spongin development.

Spicules. Robust styles only.

STYles (FIg. 8C, $\mathrm{C}_{1}$ ) straight or slightly curved, isodiametrical over much of their length with sharply pointed endings, 648-725.9-843 x 18-22.1-26 $\mu \mathrm{m}$.

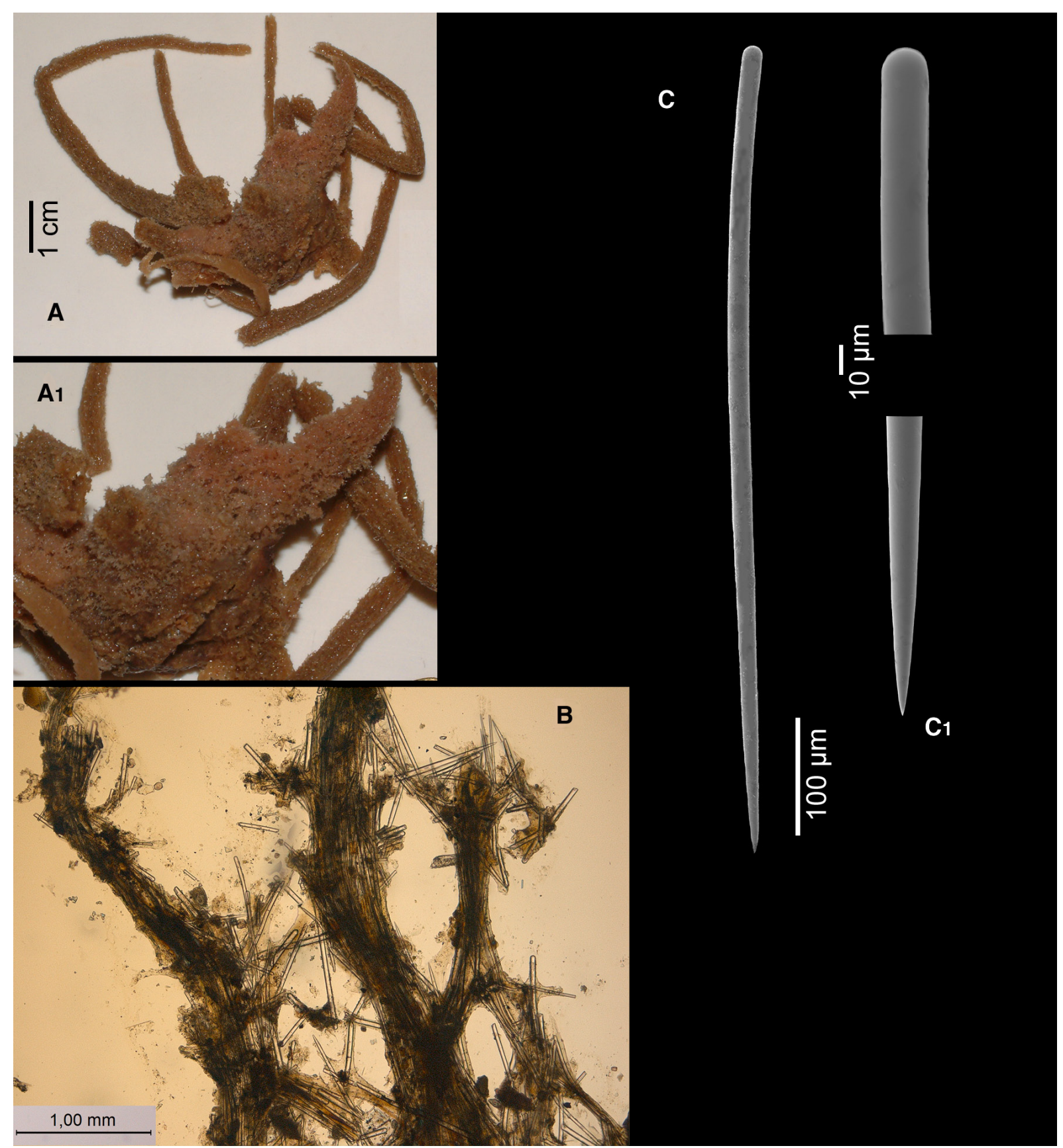

Fig. 8. Ulosa capblancensis sp. nov., Holotype ZMA Por. 09959. A. Habit. A. Detail of basal mass. B. Cross section of skeleton. C. SEM image of style. $\mathbf{C}_{1}$. Detail of apices. 


\section{Distribution and ecology}

Mauritania, on sandy platform off Cap Blanc, 10-35 m (Fig. 1, loc. 1).

\section{Remarks}

The growth form of the new species is similar in most respects to Ulosa stuposa, which was found in the same habitat. It is likely that colours differ (yellow-orange in typical U. stuposa, possibly red or redbrown in U. capblancensis sp. nov.). However, the major distinguishing features are (1) the structure of the skeleton, with weakly developed connecting fibres and thick irregular spongin-encased ascending spicule tracts, and (2) the relatively long and thick styles, on average twice or thrice as long and thick as those of U. stuposa (725 vs. $160 \mu \mathrm{m})$.

The present species might easily be confused with a local species of Dictyonella Schmidt, 1868 [possibly identical or close to Dictyonella pelligera (Schmidt, 1864)], which shares possession of erect branches and long thick styles with $U$. capblancensis sp. nov. Differences are pointed surface projections, the plumose arrangement of the spicules, and the characteristic abundance of cells with refractile granules (see Rütlzer et al. 2003) of the Dictyonella species.

\section{Discussion}

From the present new data from an area of the oceans that is relatively well-studied, it may be concluded that actual diversity of esperiopsid sponges is probably substantially underestimated. Many more species from deeper water (shelf, bathyal and abyssal habitats) along the west coasts of Africa and elsewhere may be expected to occur A problem for further discovery is the simple skeletal structure in these species, which may prevent an unambiguous description. Cases in point are literature records of Amphilectus rugosus, a Chilean species recorded from SW Africa, A. fucorum reported from disjunct localities all over the globe, or potential confusion of Ulosa species with members of halichondrid genera such as Dictyonella, Scopalina Schmidt, 1862, Stylissa Hallmann, 1914 or Hymeniacidon Bowerbank, 1858.

The distinctness of Amphilectus and Esperiopsis remains problematic, as the character differences given by Van Soest \& Hajdu (2002b) appear artificial, viz. length of styles less than $400 \mu \mathrm{m}$ and lack of microscleres other than palmate isochelae (Amphilectus), vs. longer styles and additional microscleres, sigmas, toxas and trichodragmas (Esperiopsis). The differences are also eroded by several species possessing styles with lengths in the neighbourhood of $400 \mu \mathrm{m}$ (see above). Other characters, such as the precise shape of the microscleres, like the incurved shaft of the chelae in Amphilectus utriculus sp. nov., A. fucorum and A. informis, or the architecture of the skeleton, like the more reticulate architecture of A. fucorum compared to the distinctly plumose structure observed in both Amphilectus strepsichelifer sp. nov. and Esperiopsis cimensis sp. nov., may need to be taken into account as well to arrive at a more 'natural' grouping of species of both genera. Possibly this will result in a rearrangement of species currently assigned to both genera, but support from independent datasets is needed for this.

The family Esperiopsidae, although maintained as a separate family by the latest overview of the classification (Hooper \& Van Soest 2002), was extensively emended and reduced compared to its original content (Hentschel 1923). It is founded on the lack of an ectosomal specialization, a basically plumoreticulate choanosomal skeleton and palmate isochelae, of which the latter may be either absent or complemented with sigmas. The family Mycalidae Lundbeck, 1905 is considered most closely related and many previous authors included current genera of Esperiopsidae as members of a broader concept of the family Mycalidae. However, most mycalids have a specialized ectosomal structure and the chelae are anisochelate. Relationships with desmacellid and cladorhizid sponges are also evident, but most genera of these families appear more distant. Phylogenetic relationships have not yet been seriously 
investigated and until this is addressed we refrain from proposing changes in the family content or the integrity of the genera assigned to it.

\section{Acknowledgements}

Jan Vermeulen (formerly employed by ZMA) assisted on board of RV Tyro with securing and preserving the sponges. The late Jaap van der Land invited the first author to participate in the CANCAP 7 and Mauritania II cruises. Captains and crews of HNLMS Tydemann and RV Tyro are thanked for their efforts.

\section{References}

Arnesen E. 1932. Spongia from the "Michael Sars" North Atlantic Deep-Sea Expedition 1910. Hjort J. \& Murray J. (eds) Report on the Scientific Results of the "Michael Sars" North Atlantic Deep-Sea Expedition 19103 (2): 1-29. The Trustees of the Bergen museum, Bergen. http://dx.doi.org/10.5962/ bhl.title. 11322

Bergquist P.R. \& Fromont P.J. 1988. The Marine Fauna of New Zealand: Porifera, Demospongiae. Part.4: Poecilosclerida. New Zealand Oceanographic Institute Memoir 96, New Zealand oceanographic institute, Wellington.

Burton M. 1932. Sponges. Discovery Reports 6: 237-392, pls. 48-57.

Burton M. 1940. Las Esponjas marinas del Museo Argentino de Ciencias Naturales. (Parte 1). Anales del Museo argentino de ciencias naturales 'Bernardino Rivadavia' 40 (6): 95-121, pls. I-VIII.

Burton M. 1956. The sponges of West Africa. Atlantide Report: scientific Results of the Danish Expedition to the Coasts of Tropical West Africa, 1945-1946 4: 111-147.

De Weerdt W.H. \& Van Soest R.W.M. 1986. Marine shallow-water Haplosclerida (Porifera) from the south-eastern part of the North Atlantic Ocean. Zoölogische Verhandelingen Rijksmuseum van Natuurlijke Historie te Leiden 225: 1-49.

Goodwin C., Jones J., Neely K. \& Brickle P. 2011. Sponge biodiversity of the Jason Islands and Stanley, Falkland Islands with descriptions of twelve new species. Journal of the Marine Biological Association of the United Kingdom 91 (2): 275-301. http://dx.doi.org/10.1017/S0025315410001542

Hentschel E. 1923. Erste Unterabteilung der Metazoa: Parazoa, Porifera-Schwämme. In: Kükenthal W. \& Krumbach T. (eds) Handbuch der Zoologie. Eine Naturgeschichteder Stämme des Tierreiches. Vol. 1: Protozoa, Porifera, Coelenterata, Mesozoa: 307-418, figs 288-377. Walter de Gruyter und Co, Berlin and Leipzig.

Hiemstra F. \& Van Soest R.W.M. 1991. Didiscus verdensis spec. nov. (Porifera: Halichondrida) from the Cape Verde Islands, with a revision and phylogenetic classification of the genus Didiscus. Zoölogische Mededelingen Rijksmuseum van Natuurlijke Historie te Leiden 65 (4):39-52.

Hooper J.N.A. \& Van Soest R.W.M. (eds) 2002. Systema Porifera: a guide to the classification of Sponges. Kluwer Academic/Plenum Publishers, New York.

Lendenfeld R. von 1907. Die Tetraxonia. In: Chun C. (ed.) Wissenschaftliche Ergebnisse der Deutschen Tiefsee-Expedition auf der Dampfer Valdivia 1898-1899 11 (2). G. Fisher, Jena. http://dx.doi.org/10.5962/ $\underline{\text { bhl.title. } 2171}$

Lévi C. 1952. Spongiaires de la côte du Sénégal. Bulletin de l'Institut français d'Afrique noire. Série A, Sciences naturelles 14 (1): 34-59.

Lévi C. 1956. Spongiaires de la région de Dakar. Bulletin de l'Institut français d'Afrique noire. Série A, Sciences naturelles 18 (2): 391-405. 
Lévi C. 1959. Spongiaires. In: Campagne de la 'Calypso'dans le Golfe de Guinée et aux îles Principe, São Tomé et Annobon 5: 115-141, pls. 5-6. Résultats scientifiques des Campagnes de la 'Calypso' 4. Annales de 1'Institut océanographique 37, Masson, Paris.

Lévi C. 1960. Spongiaires des côtes occidentales africaines. Bulletin de l'Institut français d'Afrique noire. Série A, Sciences naturelles 22 (3): 743-769.

Morrow C., Picton B.E., Erpenbeck D., Boury-Esnault N., Maggs C.A. \& Allcock A.L. 2012. Congruence between nuclear and mitochondrial genes in Demospongiae: A new hypoythesis for relationships within the G4 clade (Porifera: Demospongiae). Molecular Phylogenetics and Evolution 62 (1): 174-190. http:// dx.doi.org/10.1016/j.ympev.2011.09.016

Rützler K., Van Soest R.W.M. \& Alvarez B. 2003. Svenzea zeai, a Caribbean Reef Sponge with a Giant Larva, and Scopalina ruetzleri: A Comparative Fine-Structural Approach to Classification (Demospongiae, Halichondrida, Dictyonellidae). Invertebrate Biology 122 (3): 203-222. http://dx.doi. org/10.1111/j.1744-7410.2003.tb00085.x

Samaai T. \& Gibbons M. 2005. Demospongiae taxonomy and biodiversity of the Benguela region on the west coast of South Africa. African Natural History 1: 1-96.

Thiele J. 1905. Die Kiesel- und Hornschwämme der Sammlung Plate. Fauna Chiliensis III. Zoologische Jahrbücher Supplement 6: 407-496.

Topsent E. 1918. Éponges de San Thomé. Essai sur les genres Spirastrella, Donatia et Chondrilla. Archives de Zoologie expérimentale et générale 57 (6): 535-618.

Topsent E. 1928. Spongiaires de l'Atlantique et de la Méditerranée provenant des croisières du Prince Albert ler de Monaco. Résultats des campagnes scientifiques accomplies sur son yacht par Albert ${ }^{\text {er }}$ prince souverain de Monaco 74, Impr. de Monaco, Monaco.

Uriz M.J. 1988. Deep-water sponges from the continental shelf and slope off Namibia (Southwest Africa): Classes Hexactinellida and Demospongia. Monografías de Zoología Marina 3: 9-157.

Van Soest R.W.M. 1987. Biogeographic and taxonomic notes on some Eastern Atlantic sponges. In: Jones W.C. (ed.) European Contributions to the Taxonomy of Sponges: 13-28. Sherkin Island Marine Station, Sherkin Island, County Cork.

Van Soest R.W.M., Hooper J.N.A. \& Hiemstra F. 1991. Taxonomy, phylogeny and biogeography of the marine sponge genus Acarnus (Porifera: Poecilosclerida). Beaufortia 42 (3): 49-88.

Van Soest R.W.M. 1993a. Affinities of the Marine Demospongiae Fauna of the Cape Verde Islands and Tropical West Africa. Courier Forschungsinstitut Senckenberg 159: 205-219.

Van Soest R.W.M. 1993b. Distribution of sponges on the Mauritanian continental shelf. In: Wolff W.J., van der Land J., Nienhuis P.H. \& de Wilde P.A.W.J. (eds) Ecological Studies in the Coastal Waters of Mauritania. Hydrobiologia 258: 95-106.

Van Soest R.W.M., Picton B.E. \& Morrow C. 2000. Sponges of the North East Atlantic. In: World Biodiversity Database CD-ROM Series, Windows/Mac version 1.0. ETI Bioinformatics, University of Amsterdam, Amsterdam.

Van Soest R.W.M. \& Hajdu E. 2002a. Family Phellodermidae fam. nov. In: Hooper J.N.A. \& Van Soest R.W.M. (eds) Systema Porifera. A guide to the classification of sponges: 621-624.. Kluwer Academic/ Plenum Publishers, New York. 
Van Soest R.W.M. \& Hajdu E. 2002b. Family Esperiopsidae Hentschel, 1923. In: Hooper J.N.A. \& Van Soest R.W.M. (eds) Systema Porifera. A guide to the classification of sponges: 656-664.. Kluwer Academic/Plenum Publishers, New York.

Van Soest R.W.M., Beglinger E.J. \& de Voogd N.J. 2010. Skeletons in confusion: a review of astrophorid sponges with (dicho-)calthrops as structural megascleres (Porifera, Demospongiae, Astrophorida). Zookeys 68: 1-88. http://dx.doi.org/10.3897/zookeys.68.729

Van Soest R.W.M, Boury-Esnault N., Hooper J.N.A., Rützler K., de Voogd N.J., Alvarez de Glasby B., Hajdu E., Pisera A.B., Manconi R., Schoenberg C., Janussen D., Tabachnick K.R., Klautau M., Picton B., Kelly M. \& Vacelet J. 2012. World Porifera database [online]. Available from http://www. marinespecies.org/porifera [accessed 12 Feb. 2012]

Voultsiadou-Koukoura E. \& Van Soest R.W.M. 1991. Hemiasterella aristoteliana n. sp. (Porifera, Hadromerida) from the Aegean Sea with a discussion on the family Hemiasterellidae. Bijdragen tot de Dierkunde 61 (1): 43-49.

Manuscript received on: 1 May 2012

Manuscript accepted on:25 June 2012

Published on: 27 July 2012

Topic editor: Rudy Jocqué

In compliance with the $I C Z N$, printed versions of all papers are deposited in the libraries of the institutes that are members of the EJT consortium: Muséum national d'Histoire naturelle, Paris, France; National Botanic Garden of Belgium, Meise, Belgium; Royal Museum for Central Africa, Tervuren, Belgium; Natural History Museum, London, United Kingdom; Royal Belgian Institute of Natural Sciences, Brussels, Belgium; Natural History Museum of Denmark, Copenhagen, Denmark. 